Publ. RIMS, Kyoto Univ.

34 (1998), 271-276

\title{
Equivariant Maps between Representation Spheres of a Torus
}

\author{
Dedicated to Professor Teiichi Kobayashi on his 60th birthday
}

By

\begin{abstract}
Katsuhiro KOMIYA*
\end{abstract}
\section{§0. Introduction}

The Borsuk-Ulam theorem [1] states that if $f: S^{m} \rightarrow S^{n}$ is an odd map between spheres, i.e., $f(-x)=-f(x)$ for all $x \in S^{m}$, then $m \leq n$. This theorem can be extended to a class of $G$-maps $S U \rightarrow S W$ between the unit spheres of linear representations $U$ and $W$ of a compact Lie group $G$. If $G$ is a torus or a $p$-torus, i.e., if $G$ is a product of circle groups, or of cyclic groups of order $p$ with $p$ prime, then the existence of a $G$-map $f: S U \rightarrow S W$ with the fixed point set $W^{G}=\{0\}$ implies $\operatorname{dim} U \leq \operatorname{dim} W$ (see [3] and the references there).

In this paper we will see that if we make an additional assumption on $U, W$ or $f$ then $U$ must be a subrepresentation of $W$.

Let $S^{1}=\{z \in \mathbb{C}|| z \mid=1\}$ be the circle group of complex numbers with absolute value 1 . For any integer $a$ let $S^{1}$ act on $V_{a}=\mathbb{C}$ via $(z, v) \mapsto z^{a} v$ for $z \in S^{\mathrm{j}}, v \in V_{a}$. For a sequence $\left(a_{1}, \ldots, a_{k}\right)$ of integers, denote by $V\left(a_{1}, \ldots, a_{k}\right)$ the tensor product $V_{a_{1}} \otimes \cdots \otimes V_{a_{k}}$, which can be considered as a representation of the $k$-dimensional torus $T^{k}=S^{1} \times \cdots \times S^{1}$. The set of such $V\left(a_{1}, \ldots, a_{k}\right)$ gives a complete set of irreducible unitary representations of $T^{k}$, and so any finite dimensional unitary representation $U$ of $T^{k}$ decomposes into a direct sum

$$
U=\bigoplus V\left(a_{1}, \ldots, a_{k}\right)^{u\left(a_{1}, \ldots, a_{k}\right)}
$$

where $u\left(a_{1}, \ldots, a_{k}\right)$ is a nonnegative integer and $V\left(a_{1}, \ldots, a_{k}\right)^{u\left(a_{1}, \ldots, a_{k}\right)}$ denotes the direct sum of $u\left(a_{1}, \ldots, a_{k}\right)$ copies of $V\left(a_{1}, \ldots, a_{k}\right)$.

Let $\mathbb{Z}\left[x_{1}, \ldots, x_{k}\right]_{L}$ denote the ring of Laurent polynomials in $x_{1}, \ldots, x_{k}$,

$$
f\left(x_{1}, \ldots, x_{k}\right)=\sum_{i_{1}, i_{k}} a\left(i_{1}, \ldots, i_{k}\right) x_{1}^{i_{1}} \cdots x_{k}^{i_{k}},
$$

Communicated by Y. Miyaoka, November 13, 1997. Revised March 12, 1998.

1991 Mathematics Subject Classifications : 55N15, 57S99

* Department of Mathematics, Yamaguchi University, Yamaguchi 753-8512, Japan 
where $i_{1}, \ldots, i_{k}$ run over the integers $\mathbb{Z}$, and $a\left(i_{1}, \ldots, i_{k}\right)$ 's are integers and only finitely many of them are nonzero. $f\left(x_{1}, \ldots, x_{k}\right)$ is irreducible if it is not a unit and if whenever

$$
f\left(x_{1}, \ldots, x_{k}\right)=g\left(x_{1}, \ldots, x_{k}\right) \circ h\left(x_{1}, \ldots, x_{k}\right)
$$

then one of $g\left(x_{1}, \ldots, x_{k}\right)$ and $h\left(x_{1}, \ldots, x_{k}\right)$ is a unit.

Using the equivariant $K$-theory in the previous paper [2], we obtained a necessary condition for the existence of a $G$-map $S U \rightarrow S W$ in terms of the Euler classes of $U$ and $W$. Along the line of this we will do a further study for the case of $G=T^{k}$, and obtain the following results :

Theorem 0.1. Let

$$
U=\oplus V\left(a_{1}, \ldots, a_{k}\right)^{u\left(a_{1}, \ldots, a_{k}\right)}, \text { and } W=\bigoplus V\left(a_{1}, \ldots, a_{k}\right)^{w\left(a_{1}, \ldots, a_{k}\right)}
$$

be two unitary representations of $T^{k}$ with $W^{T^{k}}=\{0\}$. Assume that whenever $w\left(a_{1}, \ldots, a_{k}\right)$ is nonzero then $1-x_{1}^{a_{1} \ldots} x_{k}^{a_{k}}$ is irreducible in $\mathbb{Z}\left[x_{1}, \ldots, x_{k}\right]_{L}$. Then there exists a $T^{k}$-map $S U \rightarrow S W$ if and only if $U$ is a subrepresentation of $W$ as a real representation.

We see that $1-x_{1}^{a_{1} \cdots} x_{k}^{a_{k}}$ is irreducible if $a_{i}= \pm 1$ for some $i\left(1 \leq_{i} \leq k\right)$.

If $U$ is a unitary representation, $S^{1}$ acts on $S U$ via scalar multiplication. Then we obtain

Theorem 0.2. Let $U$ and $W$ be two unitary representations of $T^{k}$ decomposed into direct sum as in Theorem 0.1. Then there exists a $T^{k}$-map $f: S U \rightarrow S W$ such that $f(z u)=z^{m} f(u)$ for any $z \in S^{1}$ and $u \in S U$ where $m$ is a fixed nonzero integer, if and only if $u\left(a_{1}, \ldots, a_{k}\right) \leq w\left(m a_{1}, \ldots, m a_{k}\right)$ for any $\left(a_{1}, \ldots, a_{k}\right)$ with $u\left(a_{1}, \ldots, a_{k}\right) \neq 0$.

In this Theorem, if $m=1$ then $U$ must be a subrepresentation of $W$ as a complex representation.

After discussing some prerequisites in $\S 1$ and $\S 2$, we will prove Theorems 0.1 and 0.2 in $\S 3$. Finally in $\S 4$ we will correct the incorrect part of the previous paper [2].

\section{$\S 1 . G$-maps between Representation Spheres}

In this section we will recall some prerequisites from [2].

Let $R(G)$ denote the complex representation ring of a compact Lie group $G$. The Euler class $\lambda_{-1} U$ of a unitary representation $U$ of $G$ is defined by

$$
\lambda_{-1} U=\sum_{i}(-1)^{i} \Lambda^{i} U \in R(G) .
$$

where $\Lambda^{\prime} U$ is the $i$-th exterior power of $U$. The equivariant $K$-ring $K_{G}(S U)$ of the unit sphere $S U$ of $U$ is isomorphic to $R(G)$ divided by the ideal generated by $\lambda_{-1} U$ : 


$$
K_{G}(S U) \cong R(\mathrm{G}) /\left(\lambda_{-1} U\right) .
$$

For a second unitary representation $W$ of $G$, let $f: S U \rightarrow S W$ be a $G$-map. We have a commutative diagram :

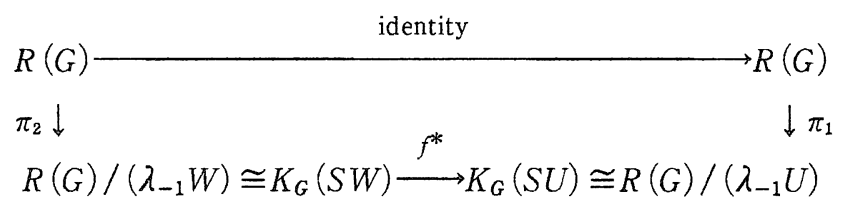

where $\pi_{1}$ and $\pi_{2}$ are the canonical projections. Then we obtain

Proposition 1.1 ([2; Proposition 2.4]). If there exists a $G-m a p S U \rightarrow S W$, then $\lambda_{-1} W \in\left(\lambda_{-1} U\right)$ in $R(G)$.

Now we restrict our attension to the $k$-dimensional torus $T^{k}$. Then

$$
R\left(T^{k}\right) \cong \mathbb{Z}\left[x_{1}, \ldots, x_{k}\right]_{L}
$$

(see [2; Proposition 3.1]). Under this isomorphism the representation $V\left(a_{1}, \ldots\right.$, $a_{k}$ ) corresponds to the monomial $x_{1}^{a_{1} \cdots} \cdot x_{k}^{a_{k}}$

Let

$$
U=\bigoplus V\left(a_{1}, \ldots, a_{k}\right)^{u\left(a_{1}, \ldots, a_{k}\right)}
$$

be a unitary representation of $T^{k}$ decomposed into a direct sum as in $\S 0$. We have in $R\left(T^{k}\right)$ or hence in $\mathbb{Z}\left[x_{1}, \ldots, x_{k}\right]_{L}$,

$$
\begin{aligned}
\lambda_{-1} U & =\prod \lambda_{-1}\left(V\left(a_{1}, \ldots, a_{k}\right)\right)^{u\left(a_{1}, \ldots, a_{k}\right)} \\
& =\prod\left(1-x_{1}^{a_{1} \cdots} x_{k}^{a_{k}}\right)^{u\left(a_{1}, \ldots, a_{k}\right)},
\end{aligned}
$$

where the product $\Pi$ is taken over the sequences $\left(a_{1}, \ldots, a_{k}\right)$.

Proposition 1.1 implies

Proposition 1.2. Let

$$
U=\oplus V\left(a_{1}, \ldots, a_{k}\right)^{u\left(a_{1}, \ldots, a_{k}\right)} \text {, and } W=\bigoplus V\left(a_{1}, \ldots, a_{k}\right)^{w\left(a_{1}, \ldots, a_{k}\right)}
$$

be two unitary representations of $T^{k}$. If there exists a $T^{k}$-map $S U \rightarrow S W$, then in $\mathbb{Z}\left[x_{1}, \ldots, x_{k}\right]_{L}$

(1.3) $\prod\left(1-x_{1}^{a_{1}} \cdots x_{k}^{a_{k}}\right)^{w\left(a_{1}, \ldots, a_{k}\right)}=\alpha\left(x_{1}, \ldots, x_{k}\right) \prod\left(1-x_{1}^{a_{1} \cdots} \cdot x_{k}^{a_{k}}\right)^{u\left(a_{1}, \ldots, a_{k}\right)}$

for some $\alpha\left(x_{1}, \ldots, x_{k}\right) \in \mathbb{Z}\left[x_{1}, \ldots, x_{k}\right] L$.

\section{§ 2. The Ring of Laurent Polynomials}

Any unit in $\mathbb{Z}\left[x_{1}, \ldots, x_{k}\right]_{L}$ is of the form $\pm x_{1}^{a_{1}} \cdots x_{k}^{a_{k}}$ for some integers $a_{1}, \ldots$, $a_{k}$. Note that $1-x_{1}^{a_{1} \cdots} x_{k}^{a_{k}}$ and $1-x_{1}^{-a_{1} \cdots} x_{k}^{-a_{k}}$ differ by a unit factor. In fact 


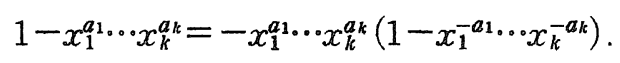

$\mathbb{Z}\left[x_{1}, \ldots, x_{k}\right]$ denotes the (ordinary) polynomial ring over, $\mathbb{Z}$, which is contained in $\mathbb{Z}\left[x_{1}, \ldots, x_{k}\right]_{L}$ as a subring. Given $f\left[x_{1}, \ldots, x_{k}\right] \in \mathbb{Z}\left[x_{1}, \ldots, x_{k}\right]_{L}$, then $x_{1}^{a_{1}} \ldots x_{k}^{a_{k}} f\left(x_{1}, \ldots, x_{k}\right)$ is in $\mathbb{Z}\left[x_{1}, \ldots, x_{k}\right]$ for sufficiently large $a_{\imath} \geq 0(1 \leq i \leq k)$. Since $\mathbb{Z}\left[x_{1}, \ldots, x_{k}\right]$ is a unique factorization domain $x_{1}^{a_{1}} \ldots x_{k}^{a_{k}} f\left(x_{1}, \ldots, x_{k}\right)$ is uniquely expressible as a product of irreducible elements up to units $(= \pm 1)$ and the order of factors, i.e.,

$$
x_{1}^{a_{1} \cdots} x_{k}^{a_{k}} f\left(x_{1}, \ldots, x_{k}\right)=f_{1}\left(x_{1}, \ldots, x_{k}\right) \cdots f_{m}\left(x_{1}, \ldots, x_{k}\right),
$$

where $f_{\imath}\left(x_{1}, \ldots, x_{k}\right)(1 \leq i \leq m)$ are irreducible polynomials in $\mathbb{Z}\left[x_{1}, \ldots, x_{k}\right]$ and are uniquely determined up to sign. The equation (2.1) gives

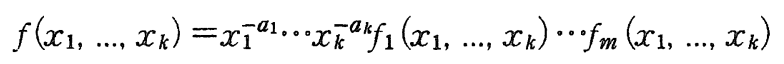

in $\mathbb{Z}\left[x_{1}, \ldots, x_{k}\right]_{L} f_{i}\left(x_{1}, \ldots, x_{k}\right)(1 \leq i \leq m)$ are also irreducible in $\mathbb{Z}\left[x_{1}, \ldots, x_{k}\right]_{L}$. This gives

Lerrma 2.2. $\mathbb{Z}\left[x_{1}, \ldots, x_{k}\right]_{L}$ is a unique factorization domain.

Lemma 2.3. (i) $1-x_{1}^{a_{1}} \cdots x_{k}^{a_{k}}$ divides $1-x_{1}^{b_{1}} \cdots x_{k}^{b_{k}}$ in $\mathbb{Z}\left[x_{1}, \ldots, x_{k}\right]_{L}$ if and only if $l\left(a_{1}, \ldots, a_{k}\right)=\left(b_{1}, \ldots, b_{k}\right)$ for some $l \in \mathbb{Z}$.

(ii) If $\left(b_{1}, \ldots, b_{k}\right) \neq(0, \ldots, 0)$ then any factorization of $1-x_{1}^{b_{1}} \cdots x_{k}^{b_{k}}$ has at most one factor of the form $1-x_{1}^{c_{1} \cdots} x_{k}^{c_{k}}\left(c_{i} \in \mathbb{Z}\right)$.

Proof. First we prove the necessity of $(i)$. This is clear if $\left(b_{1}, \ldots, b_{k}\right)=$ $(0, \ldots, 0)$. So we assume $b_{k} \neq 0$. Then we see $a_{k} \neq 0$. We assume further that $a_{k}>0$ and $b_{k}>0$. (Noting that $1-x_{1}^{c_{1} \cdots} x_{k}^{c_{k}}$ is different from $1-x_{1}^{-c_{1} \cdots} x_{k}^{-c_{k}}$ only by a unit factor, the case of $a_{k}<0$ or $b_{k}<0$ can be deduced from the case of $a_{k}>0$ and $b_{k}>0$.) Letting $m=a_{k}>0, n=b_{k}>0$ and $x=x_{k}$, then $\mathbb{Z}\left[x_{1}, \ldots, x_{k}\right]_{L}$ can be considered as the ring of Laurent polynomials in $x$ over $\mathbb{Z}\left[x_{1}, \ldots, x_{k-1}\right]_{L}$, i.e.,

$$
\mathbb{Z}\left[x_{1}, \ldots, x_{k}\right]_{L}=\mathbb{Z}\left[x_{1}, \ldots, x_{k-1}\right]_{L}[x]_{L}
$$

Letting $\mathbb{a}=\left(a_{1}, \ldots, a_{k-1}\right)$ and $\mathbb{b}=\left(b_{1}, \ldots, b_{k-1}\right)$, we put $\alpha(\mathbb{Q})=x_{1}^{a_{1}} \cdots x_{k-1}^{a_{k-1}}$ and $\alpha(\mathbb{b})=x_{1}^{b_{1} \cdots} x_{k-1}^{b_{k-1}}$. By the assumption, $1-\alpha(a) x^{m}$ divides $1-\alpha(b) x^{n}$, i.e.,

$$
1-\alpha(\mathbb{b}) x^{n}=\left(1-\alpha(\alpha) x^{m}\right)\left(\alpha_{r} x^{r}+\alpha_{r+1} x^{r+1}+\cdots+\alpha_{r+s} x^{r+s}\right),
$$

where $r, s \in \mathbb{Z}, \alpha_{r}, \alpha_{r+1}, \ldots, \alpha_{r+s} \in \mathbb{Z}\left[x_{1}, \ldots, x_{k-1}\right]_{L}, s$ is nonnegative, $\alpha_{r}$ and $\alpha_{r+s}$ is nonzero. It should be asserted here that $\alpha_{r}=1$ and $r=0$. Then (2.4) becomes

$$
1-\alpha(b) x^{n}=1+\alpha_{1} x+\cdots+\alpha_{s} x^{s}-\alpha(a) x^{m}-\alpha(a) \alpha_{1} x^{m+1}-\cdots-\alpha(a) \alpha_{s} x^{m+s} .
$$

If $s=0$, we see $m=n, \alpha(a)=\alpha(b)$ and hence $\left(a_{1}, \ldots, a_{k}\right)=\left(b_{1}, \ldots, b_{k}\right)$. If $s>0$, then we divide into the two cases: $s<m$ and $m \leq s$. For the first case, comparing the coefficients of esch $x^{l}$ on the both sides of (2.5), we see that this 
case can not occur. For the second case, comparing the coefficients again, we see that $n=m+s$, and $s$ is a multiple of $m$, say $s=(l-1) m$, then $\alpha(\boldsymbol{b})=\alpha(\boldsymbol{a})^{l}=$ $\alpha(l \boldsymbol{a})$. This implies $l\left(a_{1}, \ldots, a_{k}\right)=\left(b_{1}, \ldots, b_{k}\right)$, and completes the proof of the necessity

The sufficiency is easy. In fact, assume $l\left(a_{1}, \ldots, a_{k}\right)=\left(b_{1}, \ldots, b_{k}\right)$ and let $X=x_{1}^{a_{1} \cdots} x_{k}^{a_{k}}$. Then

$$
\begin{aligned}
1-x_{1}^{b_{1}} \cdots x_{k}^{b_{k}} & =1-X^{l} \\
& = \begin{cases}\left(1+X+X^{2}+\cdots+X^{l-1}\right)(1-X) & \text { if } l>0 \\
-X^{-1}\left(X^{l+1}+\cdots+X^{-2}+X^{-1}+1\right)(1-X) & \text { if } l<0 \\
0 & \text { if } l=0 .\end{cases}
\end{aligned}
$$

This shows the sufficiency of (i), and (ii).

\section{$\S 3$. Proof of Theorems $0.1,0.2$}

Proof of Theorem 0.1. If $U$ is a subrepresentation of $W$, then there is the inclusion map $S U \hookrightarrow S W$, which is a $T^{k}$-map.

If conversely there is a $T^{k}$-map $S U \rightarrow S W$, then we obtain the equation (1.3) from Proposition 1.2. From the assumption and Lemma 2.3 (ii) we see that $1-x_{1}^{a_{1}} \cdots x_{k}^{a_{k}}$ is irreducible if $u\left(a_{1}, \ldots, a_{k}\right)$ or $w\left(a_{1}, \ldots, a_{k}\right)$ is nonzero, and further that

$$
u\left(a_{1}, \ldots, a_{k}\right)+u\left(-a_{1}, \ldots,-a_{k}\right) \leq u^{\prime}\left(a_{1}, \ldots, a_{k}\right)+w\left(-a_{1}, \ldots,-a_{k}\right)
$$

since $\mathbb{Z}\left[x_{1}, \ldots, x_{k}\right]_{L}$ is a unique factorization domain. This means that $L$ is a subrepresentation of $W$ as a real representation, since $V\left(a_{1}, \ldots, a_{k}\right)$ and $V\left(-a_{1}\right.$, $\left.\ldots,-a_{k}\right)$ are isomorphic to each other as real representations.

For unitary representations $U, W$ of a compact Lie group $G$, and an integer $m$, let $U^{\prime}=U \otimes V_{1}$ and $W^{\prime}=W \otimes V_{m}$, where $V_{1}, V_{m}$ are the representations of $S^{1}$ given in $\S 0$. Then $U^{\prime}$ and $W^{\prime}$ become representations of $G \times S^{1}$, and we note that the following (3.1) and (3.2) are equivalent:

(3.1) There is a G-map $f: S U \rightarrow S W$ such that $f(z u)=z^{m} f(u)$ for $z \in S^{1}, u \in S U$.

(3.2) There is a $G \times S^{1}-m a p S U^{\prime} \rightarrow S W^{\prime}$.

$X * Y$ denotes the join of the topological spaces $X$ and $Y$. If $X$ and $Y$ are $G$-spaces, then $X * Y$ admits the canonical $G$-action. Two $G$-maps $f: X \rightarrow X^{\prime}$ and $g: Y \rightarrow Y^{\prime}$ canonically induce the $G$-map $f * g: X * Y \rightarrow X^{\prime} * Y^{\prime}$. For two representations $U_{1}$ and $U_{2}$ of $G$, we see $S U_{1} * S U_{2} \approx S\left(U_{1} \oplus U_{2}\right)$. So $G$-maps $h$ : $S U_{1} \rightarrow S W_{1}$ and $j: S U_{2} \rightarrow S W_{2}$ induce the $G-$ map $h * j: S\left(U_{1} \oplus U_{2}\right) \rightarrow S\left(W_{1} \oplus W_{2}\right)$.

We will now prove Theorem 0.2 .

Proof of Theorem 0.2. For unitary representations $U$ and $W$ of $T^{k}$ decomposed into direct sum as in Theorem 0.1 , representations $U^{\prime}=U \otimes V_{1}$ and 
$W^{\prime}=W \otimes V_{m}$ of $T^{k} \times S^{1}$ are decomposed as follows :

$$
U^{\prime}=\bigoplus V\left(a_{1}, \ldots, a_{k}, 1\right)^{u\left(a_{1}, \ldots, a_{k}\right)}, W^{\prime}=\bigoplus V\left(a_{1}, \ldots, a_{k}, m\right)^{w\left(a_{1}, \ldots, a_{k}\right)},
$$

where both the direct sums are taken over the sequences $\left(a_{1}, \ldots, a_{k}\right)$.

First we assume that $u\left(a_{1}, \ldots, a_{k}\right) \leq w\left(m a_{1}, \ldots, m a_{k}\right)$ if $u\left(a_{1}, \ldots, a_{k}\right) \neq 0$. The map $p: S^{1} \rightarrow S^{1}$ with $p(z)=z^{m}$ for $z \in S^{1}$ yields a $T^{k} \times S^{1}$-map from $S\left(V\left(a_{1}, \ldots\right.\right.$, $\left.\left.a_{k}, 1\right)\right)$ to $S\left(V\left(m a_{1}, \ldots, m a_{k}, m\right)\right)$. Taking the join of such $T^{k} \times S^{1}$-maps for all $\left(a_{1}, \ldots, a_{k}\right)$, we obtain a $T^{k} \times S^{1}$-map

$$
S U^{\prime}=S\left(\oplus V\left(a_{1}, \ldots, a_{k}, 1\right)^{u\left(a_{1}, \ldots, a_{k}\right)}\right) \rightarrow S\left(\oplus V\left(m a_{1}, \ldots, m a_{k}, m\right)^{u\left(a_{1}, \ldots, a_{k}\right)}\right) .
$$

This yields a $T^{k} \times S^{1}$-map $S U^{\prime} \rightarrow S W^{\prime}$, since

$$
S\left(\oplus V\left(m a_{1}, \ldots, m a_{k}, m\right)^{u\left(a_{1}, \ldots, a_{k}\right)}\right) \subset S\left(\oplus V\left(a_{1}, \ldots, a_{k}, m\right)^{w\left(a_{1}, \ldots, a_{k}\right)}\right)=S W^{\prime}
$$

by the assumption. This shows the existence of a $T^{k}$-map $S U \rightarrow S W$ with the desired property.

If conversely there is a $T^{k} \times S^{1}$-map $S U^{\prime} \rightarrow S W^{\prime}$, then from Proposition 1.2 we obtain, in $\mathbb{Z}\left[x_{1}, \ldots, x_{k}, x\right]_{L}$,

$$
\prod\left(1-x_{1}^{a_{1} \cdots} x_{k}^{a_{k}} x^{m}\right)^{w\left(a_{1}, \ldots, a_{k}\right)}=\alpha\left(x_{1}, \ldots, x_{k}, x\right) \prod\left(1-x_{1}^{a_{1} \cdots} x_{k}^{a_{k}} x\right)^{u\left(a_{1}, \ldots, a_{k}\right)}
$$

for some $\alpha\left(x_{1}, \ldots, x_{k}, x\right) \in \mathbb{Z}\left[x_{1}, \ldots, x_{k}, x\right]_{L}$, where both the products $\mathbb{I}$ are taken over the sequences $\left(a_{1}, \ldots, a_{k}\right)$. Since $1-x_{1}^{a_{1}} \ldots x_{k}^{a_{k}} x$ is irreducible, Lemmas 2.2, 2.3 imply $u\left(a_{1}, \ldots, a_{k}\right) \leq w\left(m a_{1}, \ldots, m a_{k}\right)$ if $u\left(a_{1}, \ldots, a_{k}\right) \neq 0$.

\section{§4. Correction to the Previous Paper}

Finally we should correct the previous paper [2]. On page 729 of [2] it is asserted that $L \cong \bar{U}$, but this is incorrect. If we modify the definition of $|\gamma|$ as $|\gamma|:=a_{1}+\cdots+a_{k}+b_{1}+\cdots+b_{l}$ for $\gamma=\left(a_{1}, \ldots, a_{k}, b_{1}, \ldots, b_{l}\right)$, we can still prove Theorem 1.1 of [2] with this modification of $|\gamma|$. The new proof can be done along a similar line of the previous one in $[2 ; \S 4]$.

\section{References}

[1] Borsuk, K., Drei Satze uber die $n$-dimensionale Euklidishe Sphàre, Fund. Math., 20 (1933). 177-190.

[2] Komiya, K., Equivariant $K$-theory and maps between representation spheres, Publ. RIMS, Kyoto Lniv., 31 (1995), 725-730.

[3] Marzantowicz, W., Borsuk-Ulam theorem for any compact Lie group, J. London Math. Soc. (2), 49 (1994), 195-208. 